\title{
Superradiant instability and black resonators in AdS
}

\author{
Takaaki Ishii
}

Department of Physics, Kyoto University, Kyoto 606-8502, Japan

\author{
4th International Conference on Holography, \\ String Theory and Discrete Approach \\ Hanoi, Vietnam, 2020 \\ doi:10.21468/SciPostPhysProc.4
}

\begin{abstract}
Rapidly rotating Myers-Perry-AdS black holes are unstable against rotational superradiance. From the onset of the instability, cohomogeneity-1 black resonators are constructed in five-dimensional asymptotically AdS space. By using the cohomogeneity-1 metric, perturbations of the cohomogeneity-1 black resonators are also studied.
\end{abstract}

(c) () () Copyright T. Ishii.

This work is licensed under the Creative Commons

Attribution 4.0 International License.

Published by the SciPost Foundation.
Received 01-11-2020

Accepted 17-11-2020

Published 13-08-2021

doi:10.21468/SciPostPhysProc.4.008

\section{Introduction}

In asymptotically AdS space, rotational gravitational superradiance leads to superradiant instability to rotating AdS black holes $[1,2]$. From the onset of the instability, solutions called black resonators branches off [3]. They are connected to geons in the limit of the zero horizon size. These solutions are characterized by having a helical Killing vector, where the time and rotation translations of the rotating black hole are broken to a helical one. Black resonators hence have less isometries than the stationary rotating black holes. Indeed, they are non-stationary solutions. Black resonators and geons were first constructed in four dimensions [3], where the helical Killing vector is the only continuous symmetry. Recently, by going to five dimensions and exploiting the extra symmetries in the case of equal angular momenta, black resonators and geons with cohomogeneity-1 metric have been constructed [4]. Solutions coupled to electromagnetic waves have also been obtained [5]. By making use of the cohomogeneity-1 metric, perturbations of the black resonators have been studied in [6]. A similar five-dimensional cohomogeneity-1 resonating AdS soliton geometry was also found [7]. 


\section{Cohomogeneity-1 black resonators and geons}

Cohomogeneity- 1 black resonators and geons are obtained in Einstein gravity in asymptotically global AdS space in five dimensions [4]. The metric ansatz is

$$
\begin{aligned}
d s^{2}=-\left(1+r^{2}\right) f(r) \mathrm{d} \tau^{2}+\frac{\mathrm{d} r^{2}}{\left(1+r^{2}\right) g(r)} & \\
& +\frac{r^{2}}{4}\left[\alpha(r) \sigma_{1}^{2}+\frac{1}{\alpha(r)} \sigma_{2}^{2}+\beta(r)\left(\sigma_{3}+2 h(r) \mathrm{d} \tau\right)^{2}\right],
\end{aligned}
$$

where $\sigma_{a}(a=1,2,3)$ are one-forms given by

$$
\begin{aligned}
& \sigma_{1}=-\sin \chi \mathrm{d} \theta+\cos \chi \sin \theta \mathrm{d} \phi \\
& \sigma_{2}=\cos \chi \mathrm{d} \theta+\sin \chi \sin \theta \mathrm{d} \phi \\
& \sigma_{3}=\mathrm{d} \chi+\cos \theta \mathrm{d} \phi
\end{aligned}
$$

The angular coordinates $(\theta, \phi, \chi)$ are defined in $0 \leq \theta<\pi, 0 \leq \phi<2 \pi$, and $0 \leq \chi<4 \pi$. The range of the radial coordinate $r$ is from either the origin $r=0$ (for geons) or some horizon radius $r=r_{h}$ (for black holes) to asymptotic infinity $r \rightarrow \infty$. The metric is assumed to be asymptotically $\mathrm{AdS}_{5}$ in $r \rightarrow \infty$ where $h$ approaches a constant and $f, g, \alpha, \beta \rightarrow 1$.

The Myers-Perry AdS black hole with equal angular momenta is a solution to the Einstein equations within the metric ansatz (1). It is given by

$$
\begin{aligned}
& g(r)=1-\frac{2 \mu\left(1-a^{2}\right)}{r^{2}\left(1+r^{2}\right)}+\frac{2 a^{2} \mu}{r^{4}\left(1+r^{2}\right)}, \quad \beta(r)=1+\frac{2 a^{2} \mu}{r^{4}}, \\
& h(r)=\Omega-\frac{2 \mu a}{r^{4}+2 a^{2} \mu}, \quad f(r)=\frac{g(r)}{\beta(r)}, \quad \alpha(r)=1,
\end{aligned}
$$

where the angular velocity $\Omega$ is

$$
\Omega=\frac{2 \mu a}{r_{h}^{4}+2 a^{2} \mu} .
$$

The horizon radius $r_{h}$ is given by the largest real root of $g\left(r_{h}\right)=0$. The isometry group of a general Myers-Perry black hole with independent rotations is $\mathrm{R} \times U(1) \times U(1)$, where $\mathrm{R}$ is the time translation and the $U(1)$ are for the rotations. When the two angular momenta are equal, it is enhanced to $\mathrm{R} \times U(2)$. There are $S U(2)$ angular momentum operators $L_{i}$ satisfying $\left[L_{i}, L_{j}\right]=i \epsilon_{i j k} L_{k}$, and each 1-form $\sigma_{a}$ is invariant under the $S U(2): L_{i} \sigma_{a} \equiv \mathcal{L}_{L_{i}} \sigma_{a}=0$, where $\mathcal{L}_{L_{i}}$ denotes the Lie derivative. The $U(1) \subset U(2)$ is generated by a shift of $\chi: R_{z} \equiv i \partial_{\chi}$.

This Myers-Perry AdS black hole has a gravitational superradiant instability that breaks the $U(1)$ isometry. Here we focus on a $\tau$-independent linear perturbation of the form

$$
\delta g_{\mu \nu} \mathrm{d} x^{\mu} \mathrm{d} x^{\nu}=\frac{r^{2}}{4} \delta \alpha(r)\left(\sigma_{1}^{2}-\sigma_{2}^{2}\right)=\frac{r^{2}}{2} \delta \alpha(r)\left(\sigma_{+}^{2}+\sigma_{-}^{2}\right),
$$

where we defined

$$
\sigma_{ \pm}=\frac{1}{2}\left(\sigma_{1} \mp i \sigma_{2}\right)=\frac{1}{2} e^{\mp i \chi}(\mp i \mathrm{~d} \theta+\sin \theta \mathrm{d} \phi) .
$$

This perturbation is $S U(2)$-invariant, but the terms with $\sigma_{ \pm}^{2}$ have the $U(1)$-charges \pm 2 , respectively, and hence it is not invariant under the $U(1)$ shift of $\chi: \chi \rightarrow \chi+$ const. This perturbation results in a decoupled perturbation equation for $\delta \alpha$,

$$
\delta \alpha^{\prime \prime}+\left(\frac{g^{\prime}}{g}+\frac{3+5 r^{2}}{r\left(1+r^{2}\right)}\right) \delta \alpha^{\prime}+\frac{8}{\left(1+r^{2}\right) g}\left(\frac{\beta-2}{r^{2} \beta}+\frac{2 \beta h^{2}}{\left(1+r^{2}\right) g}\right) \delta \alpha=0,
$$




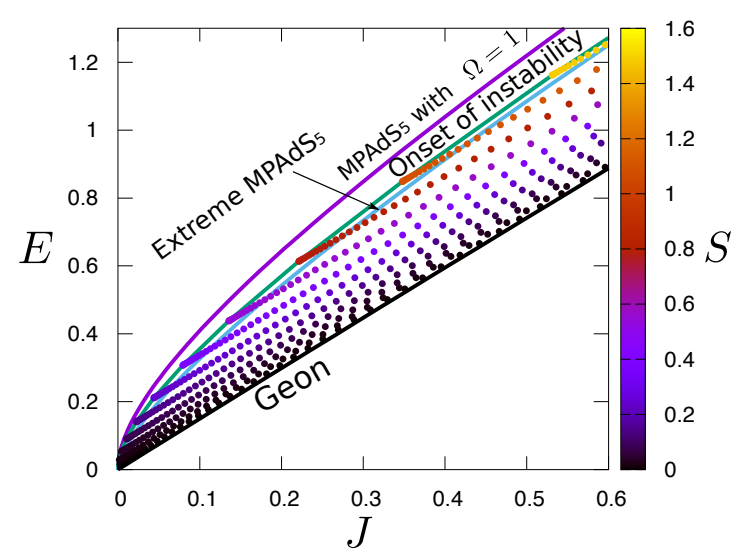

Figure 1: Phase diagram. Dots are the numerical data we constructed. The entropy $S$ is shown by the color map.

where $g$ and $\beta$ are given in (3). For fixed $r_{h}$, this equation can be solved by a nontrivial normal modes $\delta \alpha$ at a critical value of $\Omega$ that corresponds to the onset of the superradiant instability. The Myers-Perry black hole with equal angular momenta is unstable above that frequency, and the $U(1)$ isometry is broken when this instability turns up.

From the superradiant instability, black resonators with $\mathrm{R} \times S U(2)$ isometries branch off. The Einstein equations under the metric ansatz (1) can be solved for $\alpha(r) \neq 1$, and such solutions are no longer invariant under the $U(1)$. To solve the Einstein equations for black resonators, we take $h=0$ at the horizon and require $f, g, \alpha, \beta \rightarrow 1$ and $h \rightarrow \Omega$ in $r \rightarrow \infty$. These boundary conditions correspond to the rotating frame at infinity, for which we use $(\tau, \chi)$. We can switch to the non-rotating frame at infinity $(t, \psi)$ by

$$
\mathrm{d} t=\mathrm{d} \tau, \quad \mathrm{d} \psi=\mathrm{d} \chi+2 \Omega \mathrm{d} \tau .
$$

In the non-rotating frame, periodic time dependence explicitly appears in the metric as

$$
\alpha \sigma_{1}^{2}+\frac{1}{\alpha} \sigma_{2}^{2}=2\left(\alpha+\frac{1}{\alpha}\right) \bar{\sigma}_{+} \bar{\sigma}_{-}+\left(\alpha-\frac{1}{\alpha}\right)\left(e^{4 i \Omega t} \bar{\sigma}_{+}^{2}+e^{-4 i \Omega t} \bar{\sigma}_{-}^{2}\right),
$$

where $\bar{\sigma}_{a}$ are the invariant 1 -forms for the non-rotating frame at infinity by replacing $\chi$ in $\sigma_{a}$ (2) with $\psi$. The $\tau$-translation isometry $\mathrm{R}$ is interpreted to be a helical Killing vector

$$
K \equiv \frac{\partial}{\partial \tau}=\frac{\partial}{\partial t}+\Omega \frac{\partial}{\partial(\psi / 2)}
$$

The $(E, J)$ phase diagram is shown in Fig. 1. The entropy $S$ is shown by the color map. The black resonators branch off from the onset of the superradiant instability. Remarkably, they can be found even in the region where no regular Myers-Perry solutions exist. While the metric in the non-rotating frame at infinity is time dependent (9), the entropy is not. The zero horizon size limit $\left(r_{h} \rightarrow 0\right)$ of the black resonators is given by regular horizonless solutions called geons. The entropy of the black resonators approaches zero in the geon limit. The blank region below the geon curve is not covered by our ansatz.

\section{Photonic black resonators}

Cohomogeneity-1 black resonators equipped with time periodic electromagnetic waves can be obtained in Einstein-Maxwell theory. On the metric (1), we turn on a $U(1)$ gauge field as

$$
A=\gamma(r) \sigma_{1}=\gamma(r)\left(\sigma_{+}+\sigma_{-}\right) \text {. }
$$




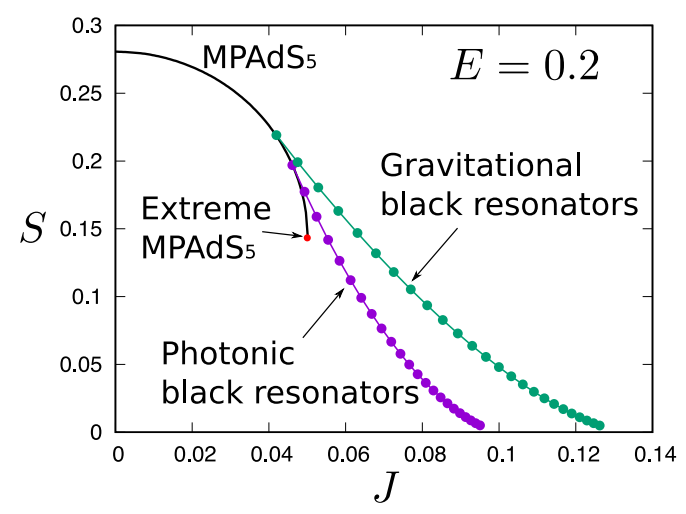

Figure 2: Entropy $S$ of the photonic black resonators for $E=0.2$. The entropies of gravitational black resonators and Myers-Perry-AdS black holes are also shown.

This gives the energy-momentum tensor consistent with the metric ansatz.

In Fig. 2, the entropies between different solutions are compared at fixed $E=0.2$. The gravitational and photonic black resonators branch off from the Myers-Perry black hole around $J=0.042$ and $J=0.046$, respectively. Both solutions can exist in the region where no regular Myers-Perry black holes can. Hence the region with regular solutions in the phase diagram is extended. However, a photonic black resonator has lower entropy than a purely gravitational black resonator at the same $(E, J)$. This implies that the former would be further unstable against $S U(2)$-symmetric perturbations and evolve into the latter if dynamical time evolution is considered.

\section{Superradiant instability of black resonators}

Black resonators obtained so far have angular frequency $\Omega>1$. In the asymptotic infinity $(r \rightarrow \infty)$, the norm of the helical Killing vector behaves as

$$
K^{2}=g_{\mu \nu} K^{\mu} K^{\nu}=g_{\tau \tau} \rightarrow-r^{2}(1-\Omega) .
$$

Thus, the Killing vector is asymptotically spacelike $K^{2}>0$ because $\Omega>1$. It has been shown that solutions with such a property should be unstable [8]. In the case of the cohomogeneity-1 black resonators, we can consider perturbations breaking the $S U(2)$ isometry of the metric.

For simplicity, we consider only the massless scalar field perturbation in the black resonator and geon backgrounds. To find the onset of instability, it is convenient to consider the eigenvalue equation of the Klein-Gordon operator as $\square \Phi=\lambda \Phi$ and search for solutions with zero eigenvalues $\lambda=0$.

The scalar field can be decomposed to modes by using the Wigner D-matrices as

$$
\Phi(\tau, r, \theta, \phi, \chi)=e^{-i \omega \tau} \sum_{|k| \leq j} \phi_{k}(r) D_{k}(\theta, \phi, \chi),
$$

where the indices of and summation over $(j, m)$ are suppressed. Using this, we obtain coupled equations for $\phi_{k}$ with the following structure,

$$
L_{k} \phi_{k}+c_{k-1} \phi_{k-2}+c_{k+1} \phi_{k+2}=0
$$




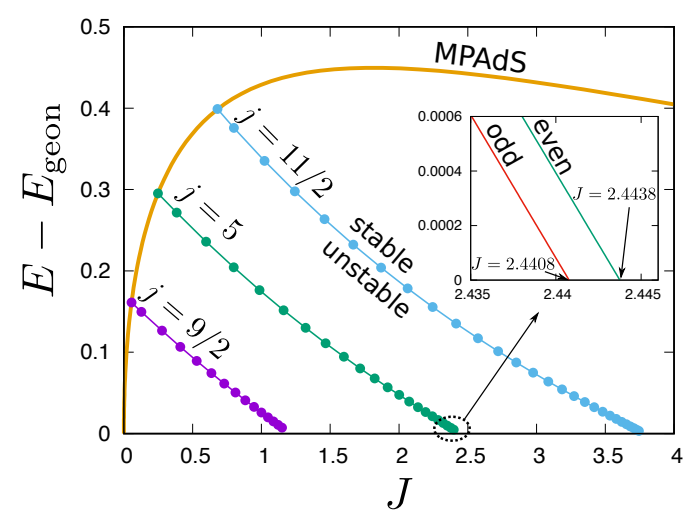

Figure 3: Phase diagram for the stability of the black resonators against a scalar field. The colored curves correspond to the onset of an instability. Black resonators are unstable to a given mode below each curve.

where

$$
\begin{aligned}
L_{k}=\left(1+r^{2}\right) g \frac{d^{2}}{d r^{2}}+\left[\frac { 1 + r ^ { 2 } } { 2 } \left(\frac{f^{\prime}}{f}+\right.\right. & \left.\left.\frac{g^{\prime}}{g}+\frac{\beta^{\prime}}{\beta}\right)+\frac{3+5 r^{2}}{r}\right] g \frac{d}{d r} \\
& -\frac{\epsilon_{k}^{2}+\epsilon_{k+1}^{2}}{r^{2}}\left(\alpha+\frac{1}{\alpha}\right)-\frac{4 k^{2}}{r^{2} \beta}+\frac{(\omega-2 k h)^{2}}{\left(1+r^{2}\right) f}-\lambda,
\end{aligned}
$$

and

$$
c_{k}=-\frac{\epsilon_{k} \epsilon_{k+1}}{r^{2}}\left(\alpha-\frac{1}{\alpha}\right) .
$$

Note that $\phi_{k}$ vanishes if $|k|>j$. If $\alpha=1$ identically such as the Myers-Perry solution, modes with different $k$ decouple. In contrast, different- $k$ modes couple in the black resonator background because the $U(1)$ isometry for the quantum number $k$ is broken. The mode coupling is "double-stepping," i.e. the mode with $k$ is coupled to those with $k \pm 2$.

We solve the coupled equations (14) and identify the instability of $\phi_{k}$ in the black resonator background. For a given $j$, we focus on the set of modes containing the $k=j$ component, which would be the mode with the highest growth rate among those with different $k$. In Fig. 3, we show the location of the onsets for the superradiant instability on black resonators for modes with $j=9 / 2, j=5$, and $j=11 / 2$. For $j=5$, there are actually two onset curves corresponding to even and odd parity modes, but they almost coincide. In the inset, we zoom into the region near the geon where it is easier to see the difference between these modes.

\section{Summary}

We studied the superradiant instability of the five-dimensional Myers-Perry black hole with equal angular momenta for $S U(2)$-invariant metric perturbations. We then constructed black resonators that branch off from the onset of the instability and are given by cohomogeneity- 1 metric. In addition to gravitational black resonators in Einstein gravity, we obtained photonic black resonators in Einstein-Maxwell theory. By using the cohomogeneity-1 black resonator as the background, we studied the perturbation of the black resonators and identified their instability. 


\section{Acknowledgments}

The work of T. I. was supported by JSPS KAKENHI Grant Number JP18H01214 and JP19K03871.

\section{References}

[1] H. K. Kunduri, J. Lucietti and H. S. Reall, Gravitational perturbations of higher dimensional rotating black holes: Tensor perturbations, Phys. Rev. D 74, 084021 (2006), doi:10.1103/PhysRevD.74.084021.

[2] K. Murata, Instabilities of Kerr-AdS $S_{5} \times S^{5}$ spacetime, Prog. Theor. Phys. 121, 1099 (2009), doi:10.1143/PTP.121.1099.

[3] O. J. C. Dias, J. E. Santos and B. Way, Black holes with a single Killing vector field: black resonators, J. High Energy Phys. 12, 171 (2015), doi:10.1007/JHEP12(2015)171.

[4] T. Ishii and K. Murata, Black resonators and geons in AdS ${ }_{5}$, Class. Quant. Grav. 36, 125011 (2019), doi:10.1088/1361-6382/ab1d76.

[5] T. Ishii and K. Murata, Photonic black resonators and photon stars in $A d S_{5}$, Class. Quant. Grav. 37, 075009 (2020), doi:10.1088/1361-6382/ab7418.

[6] T. Ishii, K. Murata, J. E. Santos and B. Way, Superradiant instability of black resonators and geons, J. High Energy Phys. 07, 206 (2020), doi:10.1007/JHEP07(2020)206.

[7] M. Garbiso, T. Ishii and K. Murata, Resonating AdS soliton, J. High Energy Phys. 08, 136 (2020), doi:10.1007/JHEP08(2020)136.

[8] S. R. Green, S. Hollands, A. Ishibashi and R. M. Wald, Superradiant instabilities of asymptotically anti-de Sitter black holes, Class. Quant. Grav. 33, 125022 (2016), doi:10.1088/0264-9381/33/12/125022. 